\title{
Surface-topography-induced enhanced transmission and directivity of microwave radiation through a subwavelength circular metal aperture
}

\author{
Matthew J. Lockyear, ${ }^{\text {a) }}$ Alastair P. Hibbins, and J. Roy Sambles \\ Thin Film Photonics Group, School of Physics, University of Exeter, Exeter EX4 4QL, United Kingdom \\ Christopher R. Lawrence \\ QinetiQ Ltd, Cody Technology Park, Farnborough, GU14 OLX, United Kingdom
}

(Received 22 December 2003; accepted 23 January 2004)

\begin{abstract}
Strongly enhanced transmission of microwave radiation $\left(\lambda_{0} \sim 5 \mathrm{~mm}\right)$ is observed through a single subwavelength circular aperture of diameter $d=2.5 \mathrm{~mm}$ in a metallic plate. The phenomenon is caused by resonant excitation of electromagnetic surface waves supported by four concentric grooves surrounding the aperture on the illuminated side of the sample. It is also shown that similar surface patterning on the output face of the sample results in very strong angular confinement (directivity) of the transmitted beam. A finite element code is used to investigate the electromagnetic fields on both the illuminated and the exit side of the structure, the predictions from which show excellent agreement with the experimental results. (C) 2004 American Institute of Physics.
\end{abstract}

[DOI: $10.1063 / 1.1688001]$

As described by standard aperture theory, ${ }^{1}$ circular holes with diameter $(d)$ smaller than the wavelength $\left(\lambda_{0}\right)$ of incident radiation are considered to possess poor transmissive properties, with the transmission normalized to the area of the hole being proportional to $(d / \lambda) .{ }^{4}$ Further, for $\lambda_{0} \gg d$ the resultant Airy disk pattern of irradiance in the far field is very broad in angle with the aperture resembling a secondary point source. However, Ebbesen et $_{\text {al. }}{ }^{2}$ have recently shown that it is in fact possible to obtain transmission of light through subwavelength hole arrays in thin metal films several orders of magnitude greater than that predicted. ${ }^{1}$ The enhanced transmission phenomenon has been associated with the excitation of electromagnetic (EM) surface modes often referred to as surface plasmons (SPs). ${ }^{3-6}$ Lezec et $a l^{7}$ have proposed a similar idea to explain enhanced transmission through a single circular subwavelength aperture, while Martin-Moreno et al. ${ }^{8}$ recently explained resonantly excited transmission of radiation through a thin slit centered within surface grooves with the same arguments. All these experiments have been in the optical regime. Here we present an experimental study at microwave frequencies of a single subwavelength aperture $(d \sim \lambda / 2)$ having a surface photonic structure comprising of four concentric grooves on either the illuminated or the exit side of the sample or both. Undertaking our experiment at these longer wavelengths allows for a precision of manufacture and of optimization that would be difficult to replicate at optical frequencies.

In the long wavelength limit the dispersion of the SP is polaritonlike since it closely follows the light line. ${ }^{9}$ The mode simply resembles a surface current, being loosely bound to a planar interface with fields extending by many wavelengths into the dielectric, and a skin depth inside the metal which is less than a micrometer. Introduction of periodic surface structure results in an increase in effective sur-

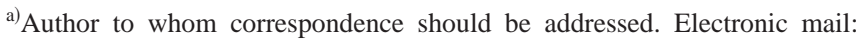
m.j.lockyear@exeter.ac.uk
}

face impedance close to the Brillouin zone boundary, ${ }^{10,11}$ hence perturbing the dispersion of the mode and increasing its degree of localization at the metal-dielectric interface. In this region of momentum space, the mode may be described as a surface plasmon.

A characteristic of grating-coupled SPs is the high-field enhancement at the interface produced by the driven oscillation of surface charge density when energy and momentum conservation criteria are met. It is these high fields that are associated with transmission enhancement through the aperture. Before fabricating the sample, the profile of the SP supporting structure was first optimized using a finite element code, ${ }^{11}$ obtaining regions of high-field intensity in the vicinity of the aperture. To further increase coupling between SPs supported on the illuminated and the exit side of the sample, the area immediately surrounding the hole was countersunk equally from each side as shown in Fig. 1 (inset), effectively reducing the depth $(h)$ of the aperture to zero

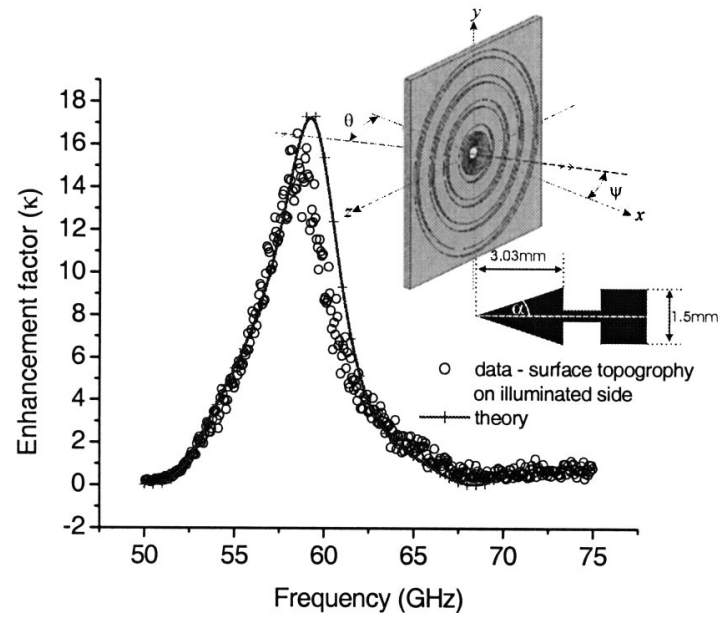

FIG. 1. Transmission enhancement factor $(\kappa)$ of a circular subwavelength aperture with surface topography on the illuminated face only (normalized to an identical aperture with no corrugation). Inset: the countersunk cross section with $\alpha=13.9^{\circ}$. 


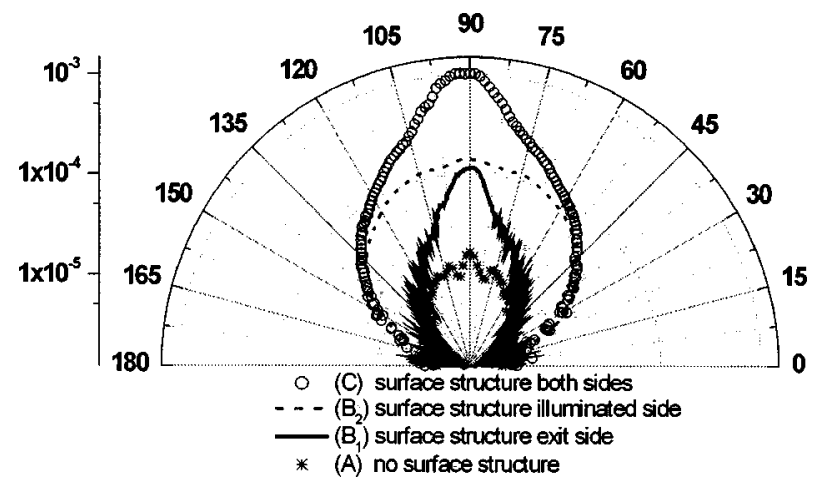

FIG. 2. Experimental angle dependent transmission spectra at the resonant frequency of each sample. (Note logarithmic scale.)

before finally optimizing for transmission using the modelling code.

Three samples were manufactured for the present study, milled into a $300 \times 300 \times 1.5 \mathrm{~mm}$ aluminum sheet. Sample $A$ is a single countersunk circular aperture $(d=2.5 \mathrm{~mm})$ with no patterning. Sample $B$ consisted of an identical aperture surrounded (on one sample face only) by four concentric rectangular grooves of depth $0.55 \mathrm{~mm}$ and width $1.50 \mathrm{~mm}$. The radii of the grooves measured $5,10,15$, and $20 \mathrm{~mm}$, respectively, from the center of the aperture to the center of each groove, providing a periodicity $\lambda_{g}=5.00 \pm 0.03 \mathrm{~mm}$. This allows an investigation of the influence of surface topography on either the exit $\left(B_{1}\right)$ or the illuminated side $\left(B_{2}\right)$ of the structure. Sample $C$ was formed by reproducing the structure in $B$ on both sides of the sample. Radiation of frequency $50<f_{0}<75 \mathrm{GHz}$ is normally incident upon the sample $\left(\theta=0^{\circ}\right)$, and polarized such that the electric vector is in the $z y$ plane (Fig. 1). The data is recorded with the signal horn positioned at a perpendicular distance of $600 \mathrm{~mm}$ from the sample, with the detector horn $350 \mathrm{~mm}$ from the exit side, mounted upon an arm which extends from a computer controlled turntable allowing the angular distribution of the transmitted signal in the plane of polarization to be measured as a function of frequency and transmission angle, $\psi$.

Figure 1 shows that the addition of the surface corrugation on the illuminated face results in a 16-fold enhancement in peak transmission over that which was observed experimentally via the "bare" countersunk aperture. Good agreement with the computer modelled predictions is also obtained. Note also that maximum enhanced transmission occurs at $\lambda_{0} \sim \lambda_{g}$, suggesting that the transmission phenomena is strongly linked to the excitation of surface waves since for normal incidence, $k_{0} \sim k_{\mathrm{SP}} \sim N k_{g}$, where $N$ is an integer and $k_{0}, k_{\mathrm{SP}}$, and $k_{g}$ are the incident, surface wave, and grating wave vector, respectively.

Figure 2 shows the abnormalized $\psi$-dependent transmission spectra on resonance of samples $A, B_{1}, B_{2}$, and $C$. It is clear that sample $B_{2}$ (orientated with concentric rings on the illuminated side only) shows a substantial increase in transmission over that recorded for the aperture with no corrugation $(A)$. In the absence of surface topography on the exit side, the transmitted signal is distributed over a wide range of $\psi$ in the transmitted half space. The forwardness that the angular distributions of radiation from samples $A$ and $B_{2}$ possess is mainly due to the ratio of hole diameter to incident Downloaded 17 Apr 2008 to 144.173.6.75. Redistribution subject

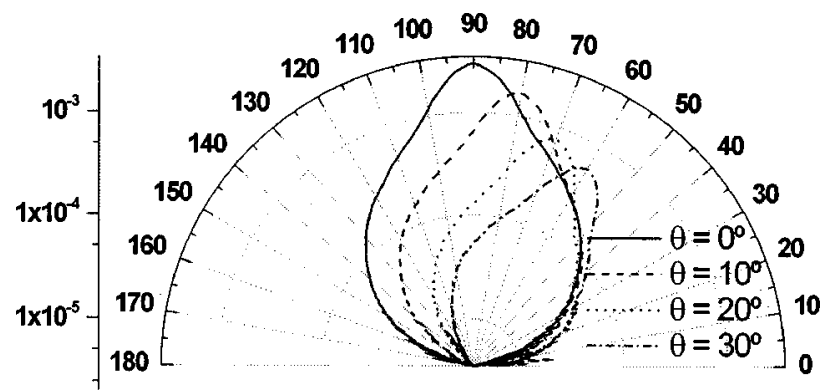

FIG. 3. Experimental angle dependent transmission spectrum of the structure having concentric rings on both surfaces, at the resonant frequency of the sample for incident angles of $0^{\circ}, 10^{\circ}, 20^{\circ}$, and $30^{\circ}$. (Note for non-normal incidence the exit face of the plate is rotated by the incidence angle relative to the direction of the incident beam which defines $-90^{\circ}$ in this figure.)

wavelength $\left(d \sim \lambda_{0} / 2\right)$, as angular-invariant diffraction would only occur for $d \ll \lambda_{0}$ (according to the HuygensFresnel principle). For a single-sided sample with concentric grating on the illuminated side only, enhanced transmission is observed with little angular confinement of the transmitted signal, with the transmission peak at resonance possessing at a width of $74^{\circ}$. The angle dependent transmission spectrum for sample $B_{1}$ (with grooves on the exit side only) demonstrates extraordinarily narrow angular distribution of the transmitted signal, possessing a transmission width of $16^{\circ}$. Furthermore, modeling the absolute transmission $(T)$ of samples $A$ and $B_{1}$ normalized to the area of the hole shows that sample $B_{1}$ exhibits no enhancement of the transmitted signal $\left(T_{A}=2.22, T_{B 1}=2.21\right)$. Thus it is clear that the concentric ring surface structure on the exit side of the sample results in collimation only, and plays no part in the enhancement of the transmitted signal.

Figure 3 shows the angle dependent transmission spectrum of the double-sided structure on resonance at $\theta=0^{\circ}$. The beaming effect from the structured exit side of the sample results in a transmitted signal which exits the aperture largely normal to the sample surface. Notice that rotating the sample such that the radiation is incident other than normal to the sample still gives an emitted beam perpendicular to the plane of the sample. Hence the concentric ring structure on the exit side of the sample not only confines the transmitted signal to a remarkably tight angular distribution but also forces the signal to exit the aperture normal to the surface plane largely irrespective of angle of incidence. In order to understand the remarkable properties of the samples under study it is useful to evaluate the modeled EM fields obtained from the finite element code in both the incident and transmitted half spaces. Figure 4 shows (a) the time averaged magnetic field $(H)$ magnitude, and (b) the instantaneous electric field $(E)$ strength along a line through the center of the aperture lying in the plane of polarization at a phase corresponding to maximum enhancement. Also shown is the Poynting vector distribution close to the surface of the double sided structure.

From comparison of the instantaneous fields at maximum enhancement it is found that the $E$ and $H$ fields near the metal surface are separated by approximately $90^{\circ}$ in phase with respect to each other both spatially and temporally, a standing wave characteristic. However, unlike a true standing wave the Poynting vector shows a small but finite net power flow across the surface of the substrate towards the to AIP license or copyright; see http://apl.aip.org/apl/copyright.jsp 

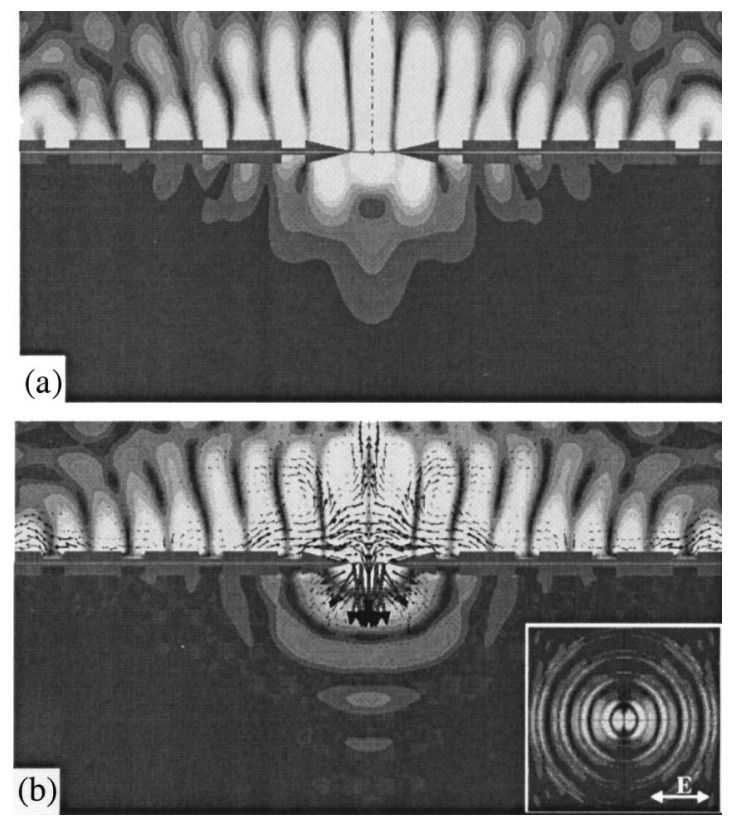

FIG. 4. (a) The modeled time-averaged magnetic field strength and (b) the instantaneous electric field strength at a phase corresponding to maximum enhancement of the $E$ fields. Also shown is the Poynting vector distribution close to the surface of the structure having concentric surface ring patterning on both sides, when radiation is normally incident from above. Inset: time averaged electric field strength at the surface of the sample in the $y z$ plane, and the orientation of the incident electric vector.

aperture. Both (a) and (b) show enhancement of the fields on the illuminated side of the sample, with maximum enhancement (25-fold increase of electric field magnitude) occurring at the sharp ring formed by countersinking at the aperture entrance. Note also that the surface wave is not a radial wave, but is a standing wave with maximum field magnitude in the plane of polarization. The two dimensionality of the surface wave is shown in Fig. 4(b)(inset); with the time averaged electric field strength of the electric field over the $y z$ plane. The standing wave supported by the structure results in a redistribution of energy, such that regions of high energy density are located in the vicinity of the hole (Fig. 4), hence increasing the power flow through the aperture compared to the bare hole geometry.

Figures 4(a) and 4(b) clearly show enhancement of the $E$ and $H$ fields, respectively, up to $16 \mathrm{~mm}$ from the substrate in the lower half space. These enhancements in field magnitude occur with the same regular spacing and position relative to the surface corrugation as those in the upper half space, al- though reduced in magnitude. This shows that the optimum geometry to in-couple plane wave radiation is also the most efficient to out-couple. Furthermore, it is clear that there exist a number of regions of relatively high field enhancement on the exit side of the sample, whereby each region may reradiate power and as such be considered as secondary sources. It is these secondary sources in combination with the primary source (the aperture) that results in the multiple source interference pattern responsible for the strong angular confinement and directivity of the transmitted signal. As the position and relative magnitude of these sources are constant with respect to each other as a function of incident angle, the transmitted signal is emitted normal to the surface of the sample.

In summary, we have presented a study of enhanced transmission through a subwavelength aperture surrounded by a photonic surface in the microwave regime. A remarkable 16-fold enhancement of transmission is observed experimentally and is verified by computer modeling. This is attributed to a near-standing wave supported at the surface of the structure on the illuminated side of the sample which increases the energy density close to the hole. Furthermore, the concentric ring patterning of the exit surface leads to strong angular confinement of the exit beam and a directivity that is largely independent of the angle of incidence.

The authors are grateful for support from the Engineering and Physical Sciences Research Council and the provision of a CASE award by QinetiQ (Farnborough) for M.J.L. This work was carried out as part of Technology Group 09 of the MoD Corporate Research Fund.

${ }^{1}$ H. A. Bethe, Phys. Rev. 66, 163 (1944).

${ }^{2}$ T. W. Ebbesen, H. J. Lezec, H. F. Ghaemi, T. Thio, and P. A. Wolff, Nature (London) 391, 667 (1998).

${ }^{3}$ J. R. Sambles, Nature (London) 391, 641 (1998).

${ }^{4}$ H. F. Ghaemi, T. Thio, D. E. Grupp, T. W. Ebbesen, and H. J. Lezec, Phys. Rev. B 58, 6779 (1998).

${ }^{5}$ E. Popov, M. Neviere, S. Enoch, and R. Reinisch, Phys. Rev. B 62, 16100 (2000).

${ }^{6}$ L. Martin-Moreno, F. J. Garcia-Vidal, and H. J. Lezec, Opt. Commun. 200, 1 (2001).

${ }^{7}$ H. J. Lezec, A. Degiron, E. Devaux, R. A. Linke, L. Martin-Moreno, F. J. García-Vidal, and T. W. Ebbesen, Science 297, 820 (2002).

${ }^{8}$ L. Martin-Moreno, F. J. García-Vidal, H. J. Lezec, A. Degiron, and T. W. Ebbesen, Phys. Rev. Lett. 90, 167401 (2003).

${ }^{9}$ H. Raether, Surface Plasmons (Springer, Berlin, 1988).

${ }^{10}$ D. Sievenpiper, L. Zhang, R. F. J. Broas, N. G. Alexopolous, and E. Yablonovitch, IEEE Trans. Microwave Theory Tech. 47, 11 (1999).

${ }^{11}$ Ansoft Corporation, Pittsburgh, PA (www.ansoft.com). 